

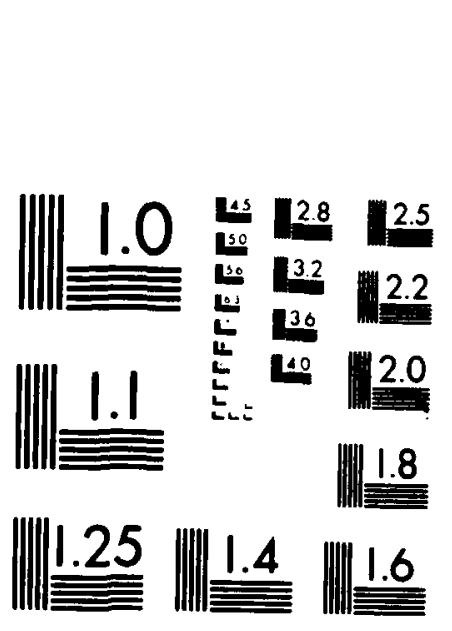

MICROCOPY RESOLUTION TEST CHART NaTINNAL BUREAL OF STANDARTS. 1963 A 


\section{$\because$ OISE-EILE LUEL}

REPORT SD-TR-87-39

\section{A GaAs MESFET Balanced Mixer with Very Low Intermodulation}

\author{
S. A. MAAS \\ Electronics Research Laboratory \\ Laboratory Operations \\ The Aerospace Corporation \\ El Segundo, CA 90245
}

31 August 1987

\author{
Prepared for \\ SPACE DIVISION \\ AIR FORCE SYSTEMS COMMAND \\ Los Angeles Air Force Station \\ P.O. Box 92960, Worldway Postal Center \\ Los Angeles, CA 90009-2960
}

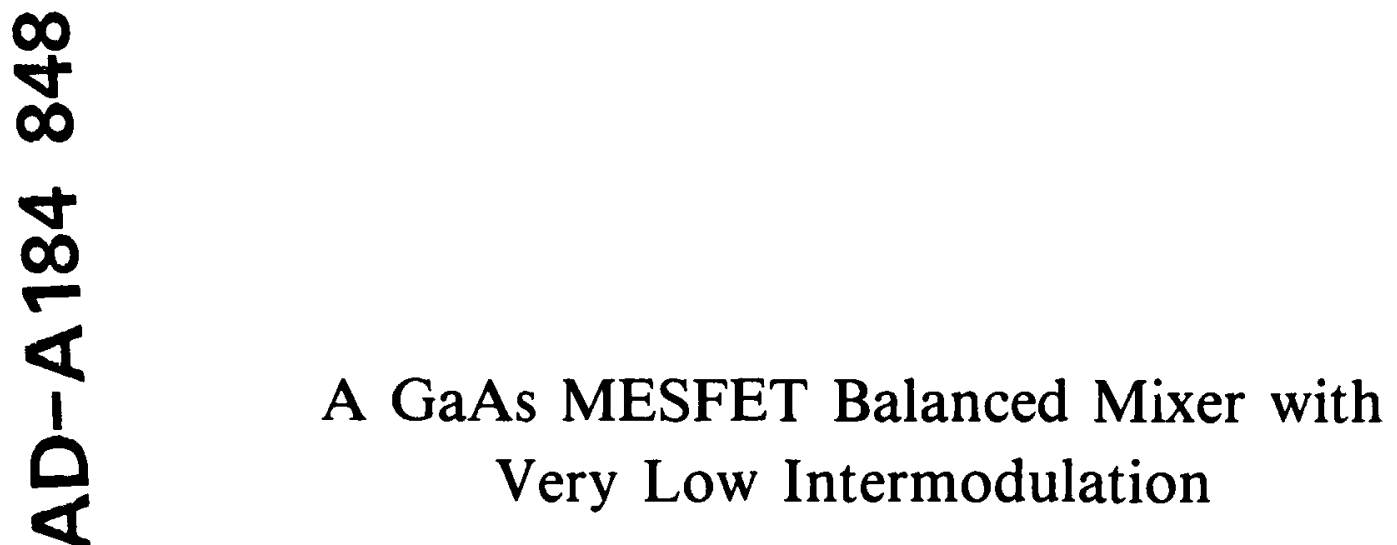

APPROVED FOR PUBLIC RELEASE:

DISTRIBUTION UNLIMITED

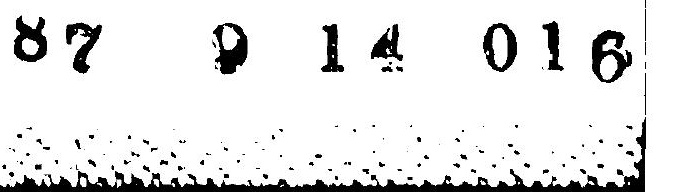


This report was submitted by The Aerospace Corporation, El Segundo, CA 90245, under Contract No. F04701-85-C-0086-P00016 with the Space Division, P. O. Box 92960, Worldway Postal Center, Los Angeles, CA 90009-2960. It was reviewed and approved for The Aerospace Corporation by $M$. J. Daugherty, Director, Electronics Research Laboratory.

Capt Richard J. Young, SD/CWX, was the project officer for the Mission-Oriented Investigation and Experimentation (MOIE) Program.

This report has been reviewed by the Public Affairs office (PAS) and is releasable to the National Technical Information Service (NTIS). At NTIS, it will be available to the general public, including foreign nationals.

This technical report has been reviewed and is approved for publication. Publication of this report does not constitute Air Force approval of the report's findings or conclusions. It is published only for the exchange and stimulation of ideas.
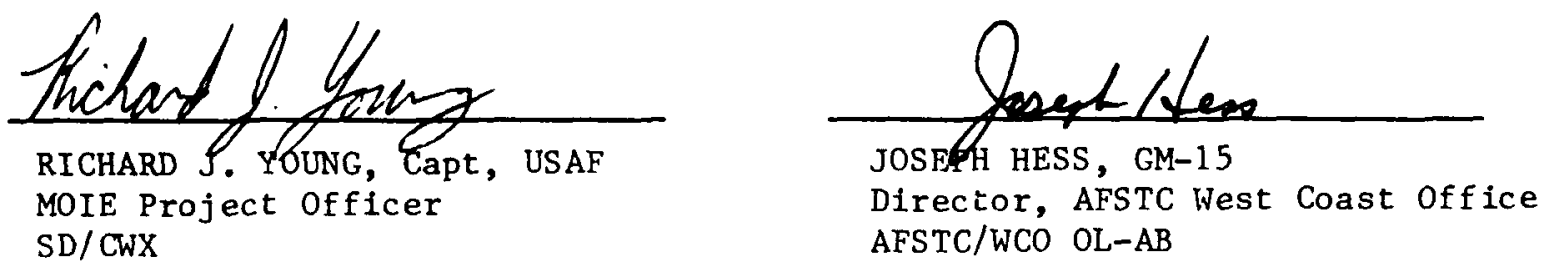


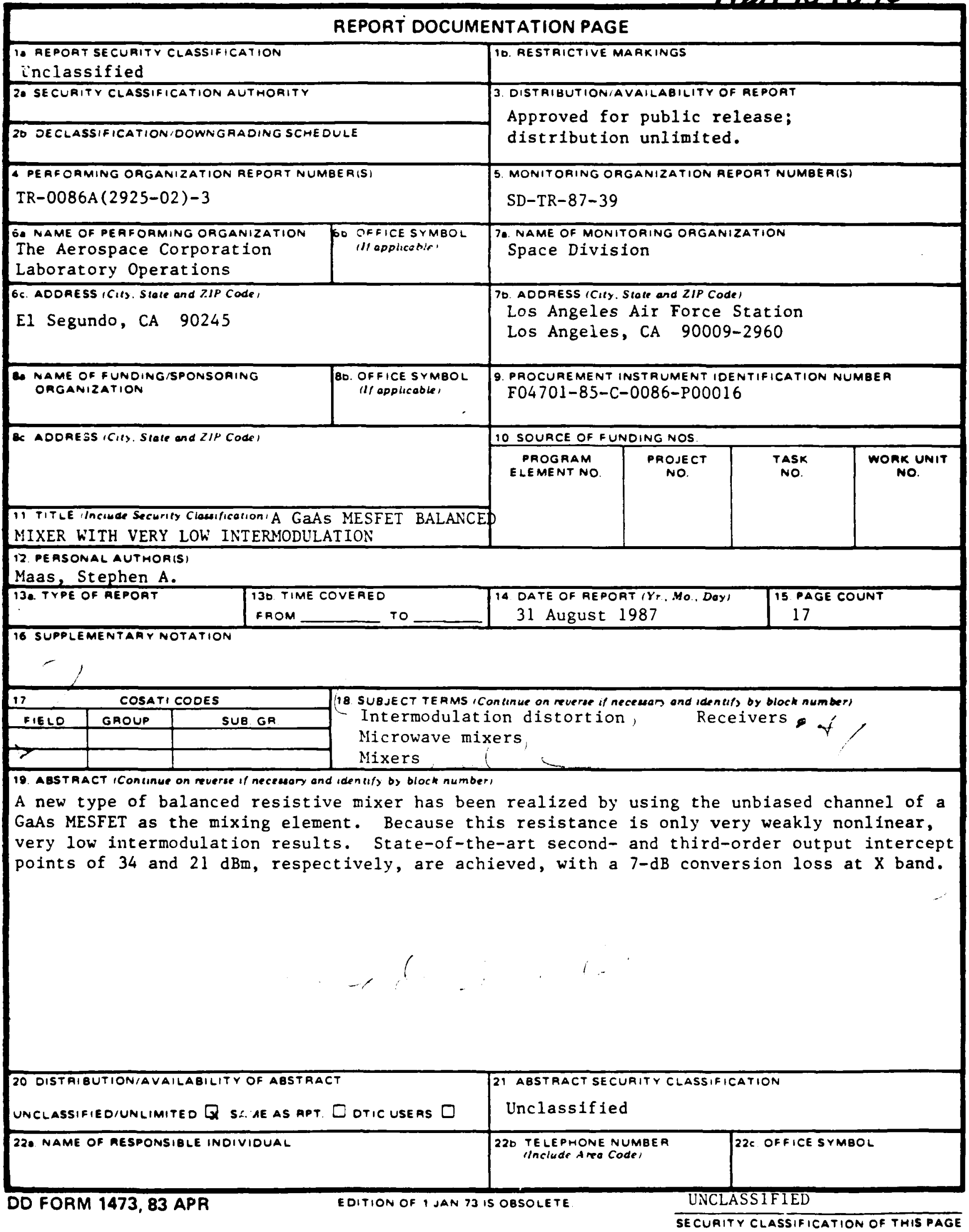


CONTENTS

I. INTRODUCTION . . . . . . . . . . . . . . . . . . 3

II. CIRCUIT DESIGN AND DESCRIPTION . . . . . . . . . . . . . 5

III. PERFORMANCE . . . . . . . . . . . . . . . . . . . . . . 9

IV. CONCLUSION . . . . . . . . . . . . . . . . . . . . 15

REFERENCES . . . . . . . . . . . . . . . . . . . . . . . 17

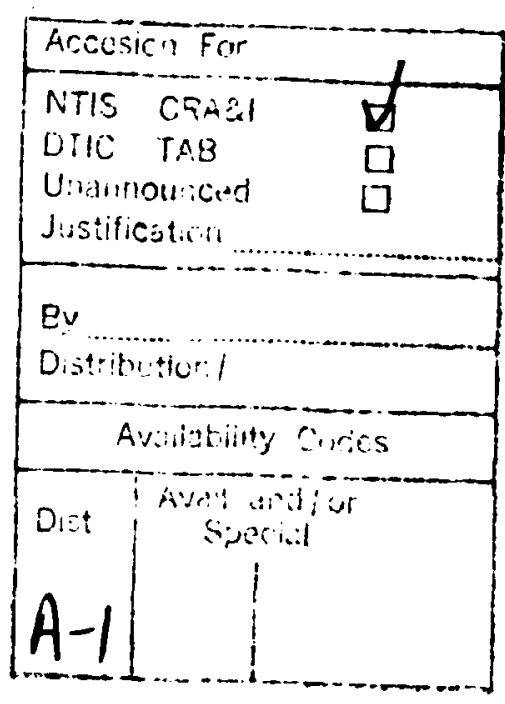


FIGURES

1. Equivalent Circuit of a GaAs MESFET with No Bias Applied

to the Drain. . . . . . . . . . . . . . . . . . . . . 6

2. Circuit of the Balanced Mixer . . . . . . . . . . . . . . . . . . 7

3. Mixer Passband with a Fixed IF Frequency and with a Fixed LO Frequency . . . . . . . . . . . . . . . . . . . . . . . . 10

4. Conversion Loss as a Function of LO Power and Gate Bias . . . . . 11

5. IM Level as a Function of LO Power and Gate Bias. . . . . . . . . 12

6. Linear, Second-Order, and Third-Order IM Levels vs. RF Leve1. . . 13 


\section{INTRODUCTION}

The Schottky-barrier diode used in most microwave mixers is one of the most strongly nonlinear devices found in microwave components; thus it is not surprising that diode mixers have relatively poor intermodulation (IM) and spurious-response properties. Methods to improve the IM performance of diode mixers have been proposed periodically, ${ }^{1-4}$ but because of practical limitations, none have been widely accepted. However, it is not necessary to use a nonlinear device for a mixer; it is theoretically possible to realize an intermodulation-free mixer by using a time-varying linear resistor.

The mixer described here approximates a time-varying linear resistor, and achieves IM performance that far exceeds the state of the art for diode mixers. The resistance that realizes the mixing element is the very weakly nonlinear resistive channel of a GaAs MESFET; one makes this resistance time-variable by applying the local oscillator (LO) to the gate. Additionally, the mixer circuit is highly practical, simple, and easy to design, and has good conversion loss and good port impedances. 


\section{CIRCUIT DESIGN AND DESCRIPTION}

Figure 1 shows the equivalent circuit of a MESFET without drain bias. The channel conductance $g\left(V_{g}\right)$ is weakly nonlinear, and is directly controlled by the internal gate/source voltage $v_{g}$. At zero drain voltage the gate-depletion capacitance is divided equally between the gate-source capacitance $C_{g s}$ and the gate-drain capacitance $C_{g d}$. The gate, drain, and source resistances, $R_{g}, R_{d}$, and $R_{s}$, respectively, are the same as in the biased device.

To realize a mixer, one applies the $L O$ and $d c$ bias to the gate, and RF to the drain. The IF output is filtered from the drain. The large value of $\mathrm{C}_{\mathrm{gd}}$ is a potential problem, however: first, because it reduces $\mathrm{RF} / \mathrm{LO}$ isolation, and second, because it couples Lo voltage to the drain and RF voltage to the gate. The lo voltage coupled to the drain swings the drain voltages over a more strongly nonlinear range, exacerbating IM generation, and the RF coupled to the gate may also increase IM as a result of transconductance nonlinearities. Therefore, in order to optimize IM performance, the LO is short-circuited at the drain, and the RF is short-circuited at the gate. The short circuit can be realized by the use of filters or a balanced structure.

The mixer circuit is shown in Fig. 2. It was designed to operate at $10 \mathrm{GHz}$ with an IF of 10 to $100 \mathrm{MHz}$. The two FETs (packaged Avantek type AT10650) were chosen from the same processing lot of devices, and were checked to ascertain that their I/V characteristics were well matched. The FET gates must be driven with a 180-degree LO phase difference; this phase split is achieved by means of a loop of transmission line 180 degrees long. The drains are in parallel at the RF, and are fed through a quarter-wave transformer that matches the 50-ohm RF input to the 25-ohm input impedance of the parallel channels. The small reactive part (due to $C_{g d}$ ) of the input impedance is tuned out empirically. The If currents in the channels are 180 degrees out of phase, and are therefore combined with a miniature RF transformer (Mini-Circuits type T4-1). The IF transformer also applies a near-optimum IF load impedance of $100 \mathrm{ohms}$ to 


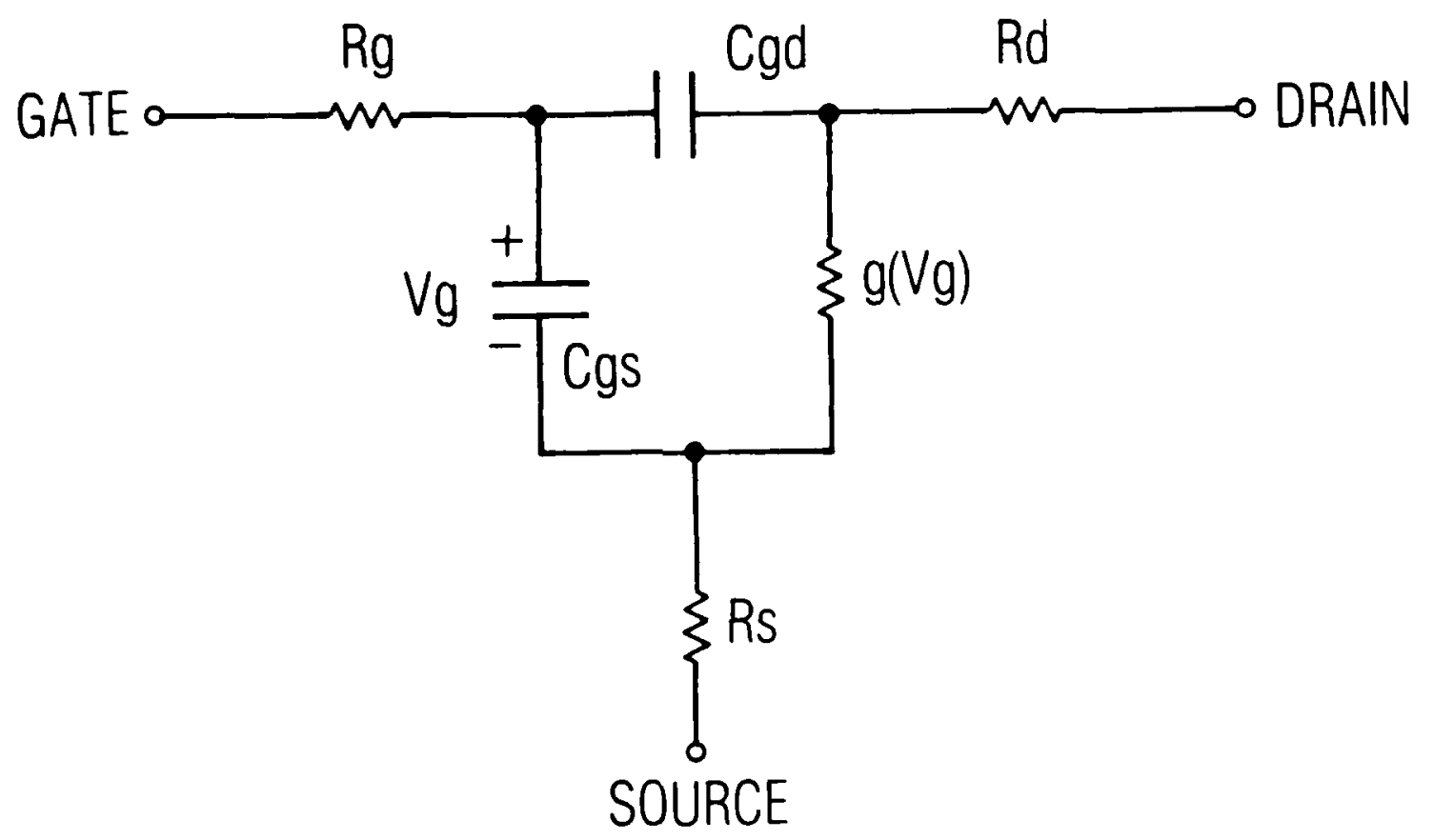

Fig. 1. Equivalent Circuit of a GaAs MESFET with No Bias Applied to the Drain 


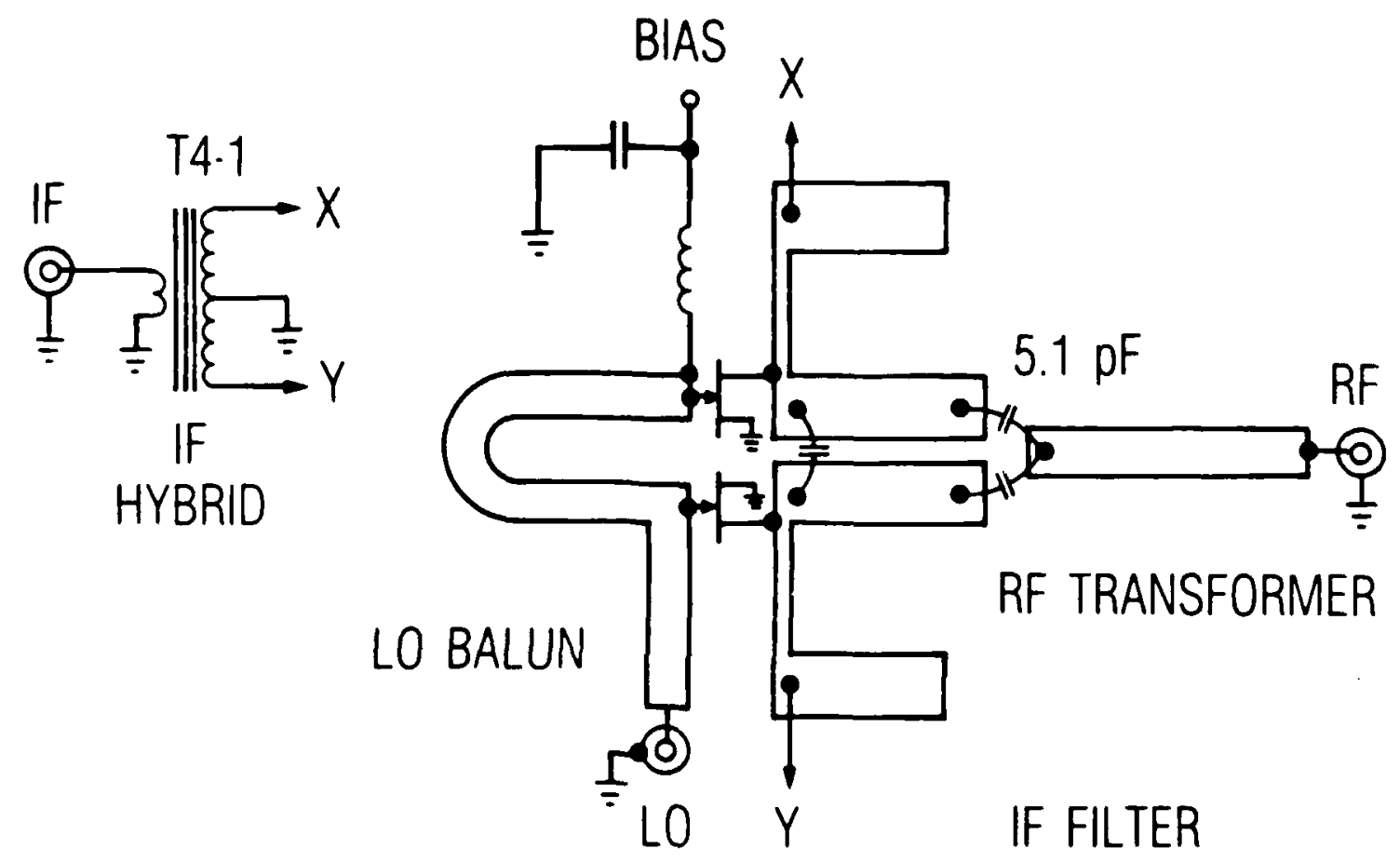

Fig. 2. Circuit of the Balanced Mixer. All capacitors are $5.1 \mathrm{pF}$. 
each FET; its loss is approximately $1 \mathrm{~dB}$ at $100 \mathrm{MHz}$.

The geometry of the circuit supplies the desired RF and Lo short circuits to the gate and drain. Because of the 180-degree phase difference of the $L$ currents through $C_{g d}$ in the two FETs, the drain node is a virtual ground to the LO. Similarly, the lo balun presents a short circuit to even-mode inputs such as the RF leakage. Many commonly used baluns, such as the Marchand balun used in doubly balanced diode mixers, may not be suitable here, because they present an open circuit to even-mode inputs. 


\section{PERFORMANCE}

Figures $3 \mathrm{a}$ and $3 \mathrm{~b}$ show the passband of the mixer for fixed IF and fixed LO. At a fixed IF frequency, conversion loss averages $7.0 \mathrm{~dB}$ over a 1-GHz band, including the 1-dB loss of the IF hybrid. The shape of the IF passband is determined primarily by the IF transformer. This performance is as good as that of most commercially available balanced-diode mixers.

Figure 4 shows the conversion loss as a function of LO power and gate bias, and Fig. 5 shows the corresponding IM levels. Higher gate bias gives better IM performance, although at lower LO power levels it is better to use somewhat lower bias. Lower bias also reduces the sensitivity of conversion loss to LO power. The sensitivity of both conversion loss and IM level to LO level is significantly lower than that of most commercially available diode mixers.

Figure 6 shows the second-and third-order IM levels as a function of RF level (the second-order component is the IF output at the difference between the two input frequencies). The second-order component exhibits the expected $2 \cdot d B / d B$ slope; however, the third-order output has a slope of $2.6 \mathrm{~dB} / \mathrm{dB}$ at low $\mathrm{RF}$ levels and rises monotonically at high input levels. This result is not unusual, and indicates that nonlinearities of degree higher than three contribute significantly to third-order IM generation. Under these circumstances it is difficult to define an intermodulation intercept point, but over this range of power levels the IM level is lower than would be indicated by a third-order intercept point at a $21-\mathrm{dBm}$ output. The second-order output intercept point from Fig. 6 is $34 \mathrm{dBm}$. 


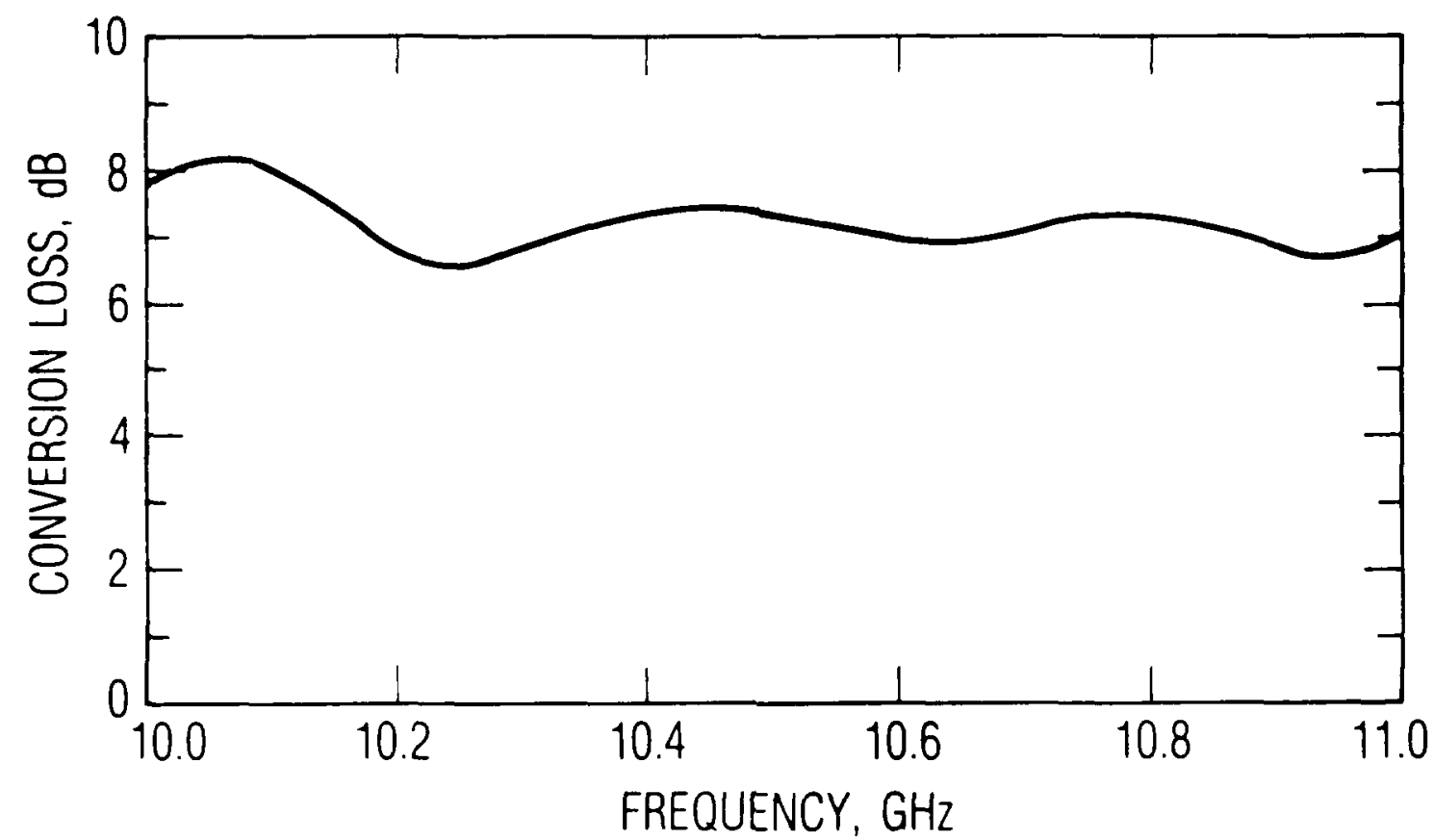

A

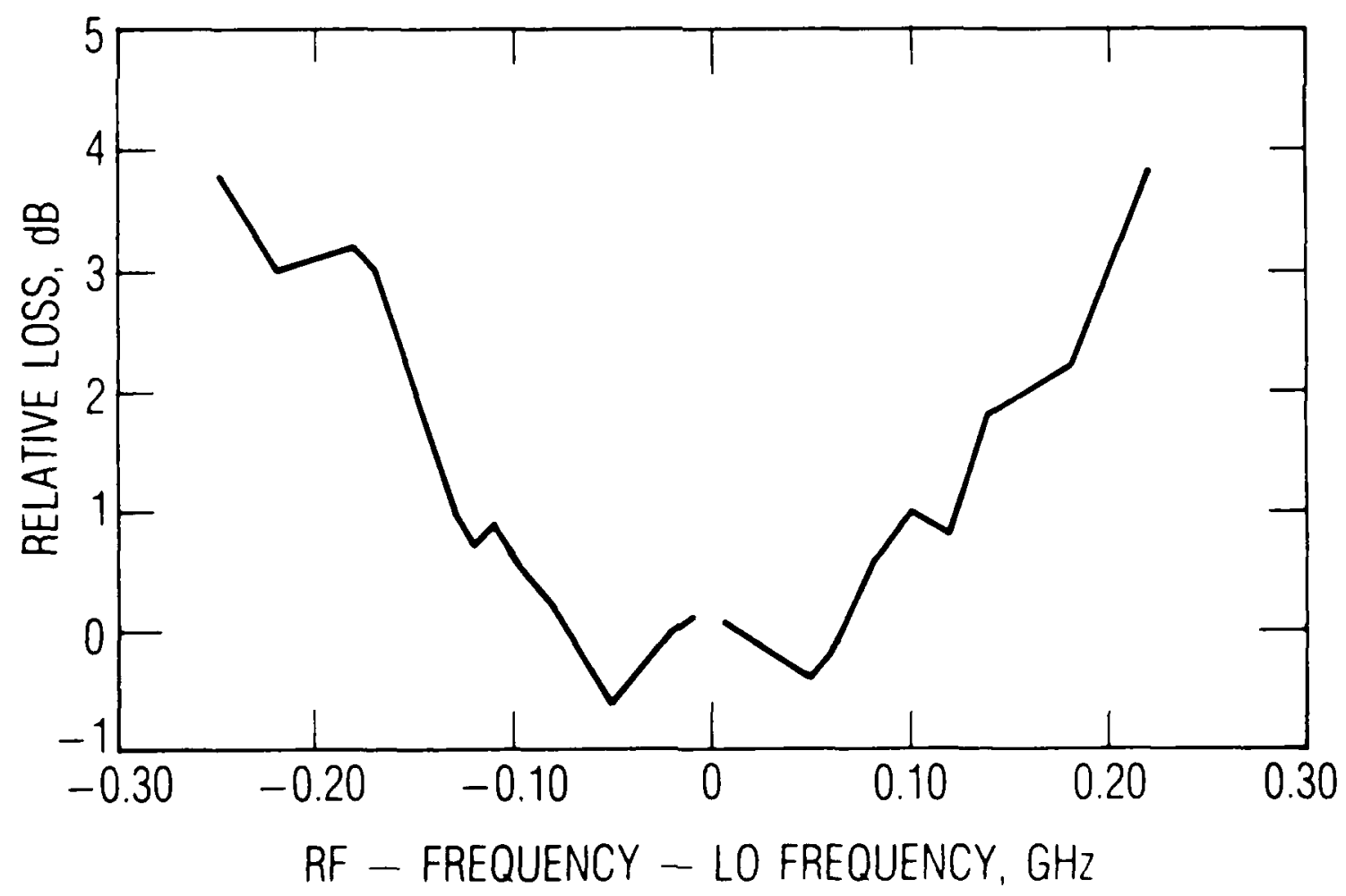

B

Fig. 3. Mixer Passband (a) with a Fixed IF Frequency and (b) with a Fixed Lo Frequency 


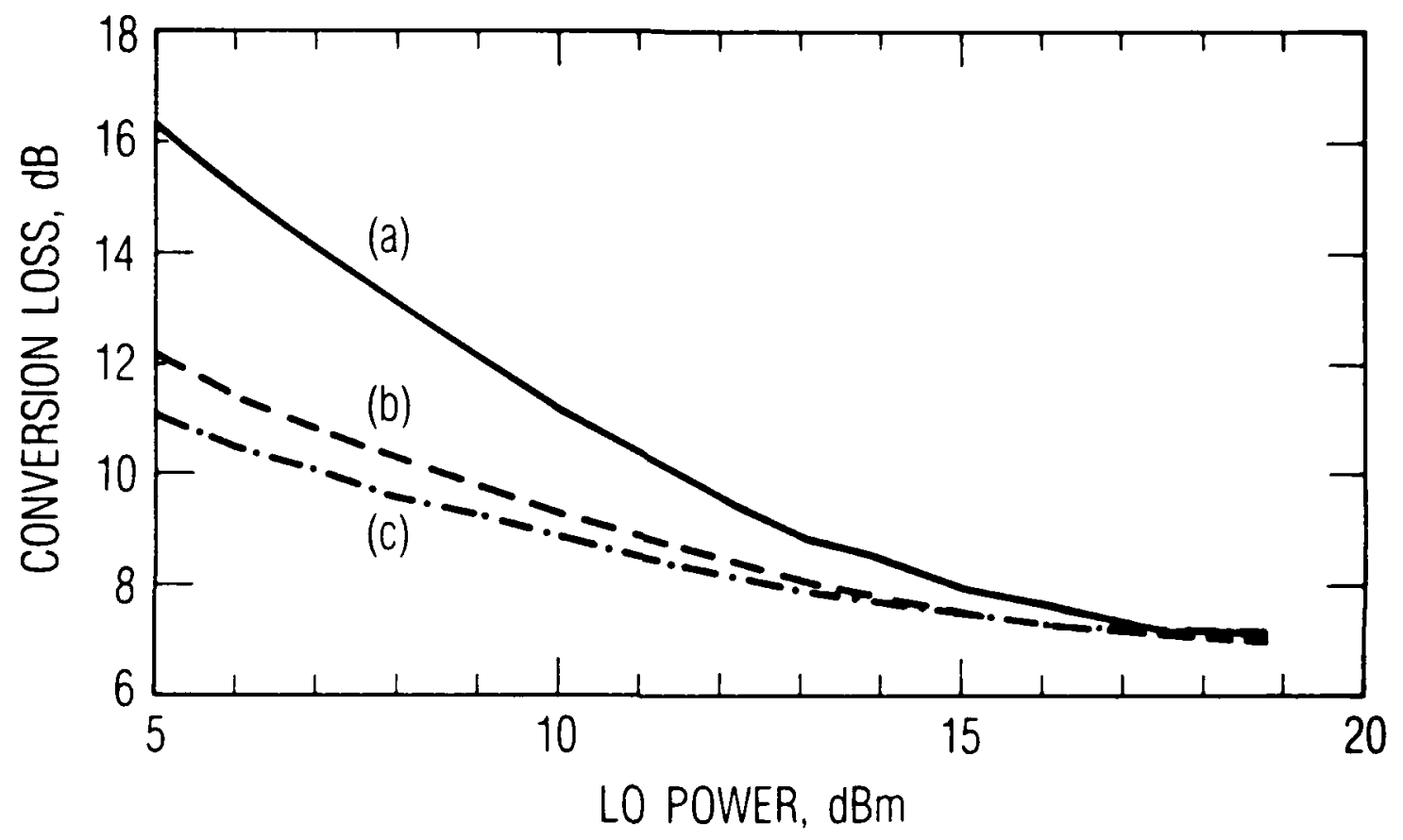

Fig. 4. Conversion Loss as a Function of LO Power and Gate Bias. (a) $\mathrm{V}_{\mathrm{gs}}=-1.10$. (b) $\mathrm{v}_{\mathrm{gs}}=-0.84$.
(c) $\mathrm{V}_{\mathrm{gs}}=-0.63$. 


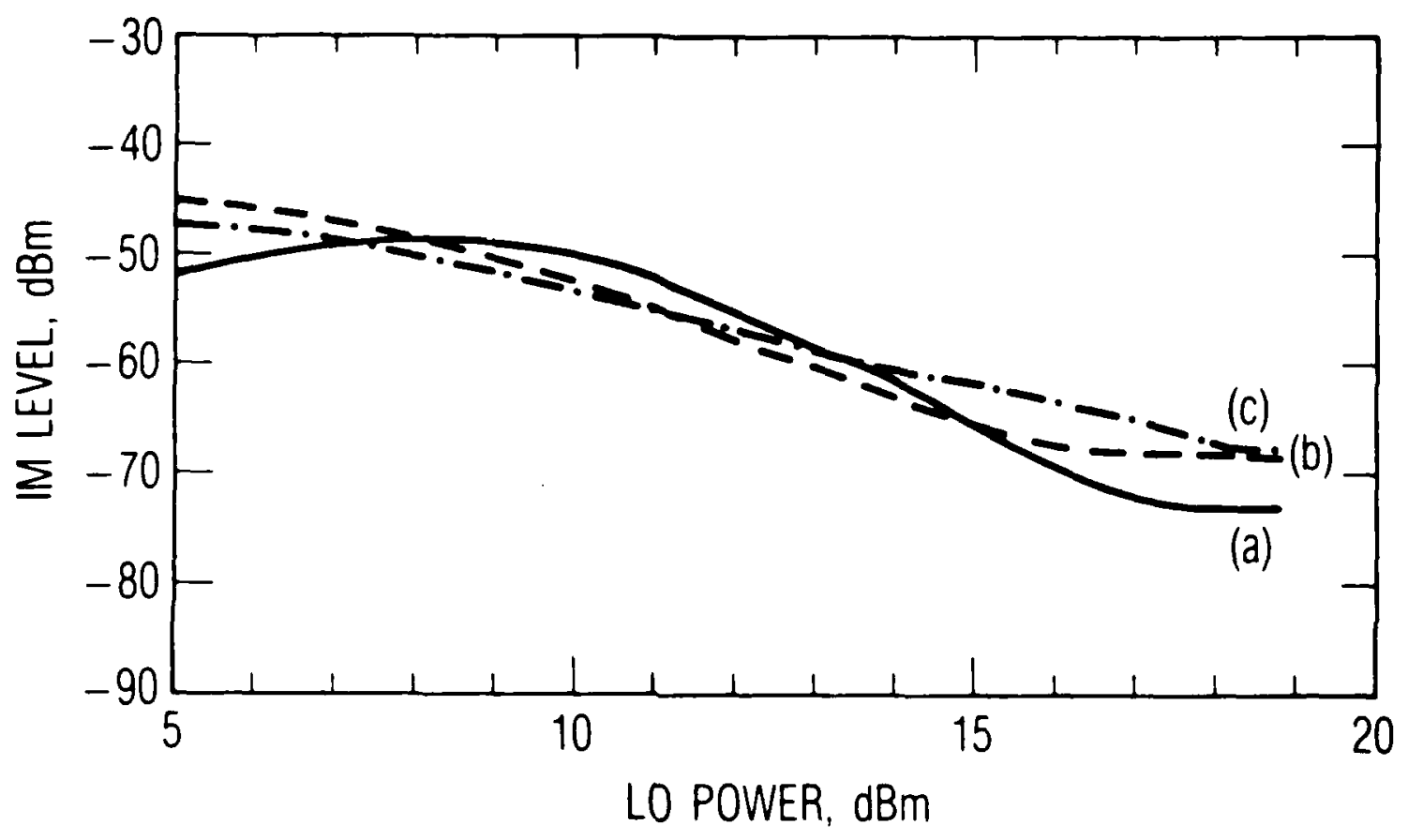

Fig. 5. IM Level as a Function of LO Power and Gate Bias. $\mathrm{RF}$ level is $-3 \mathrm{dBm}$. (a) $\mathrm{v}_{\mathrm{gs}}=-1.10$. (b) $\mathrm{v}_{\mathrm{gs}}=$
-0.84 . (c) $\mathrm{v}_{\mathrm{gs}}=-0.63$. 


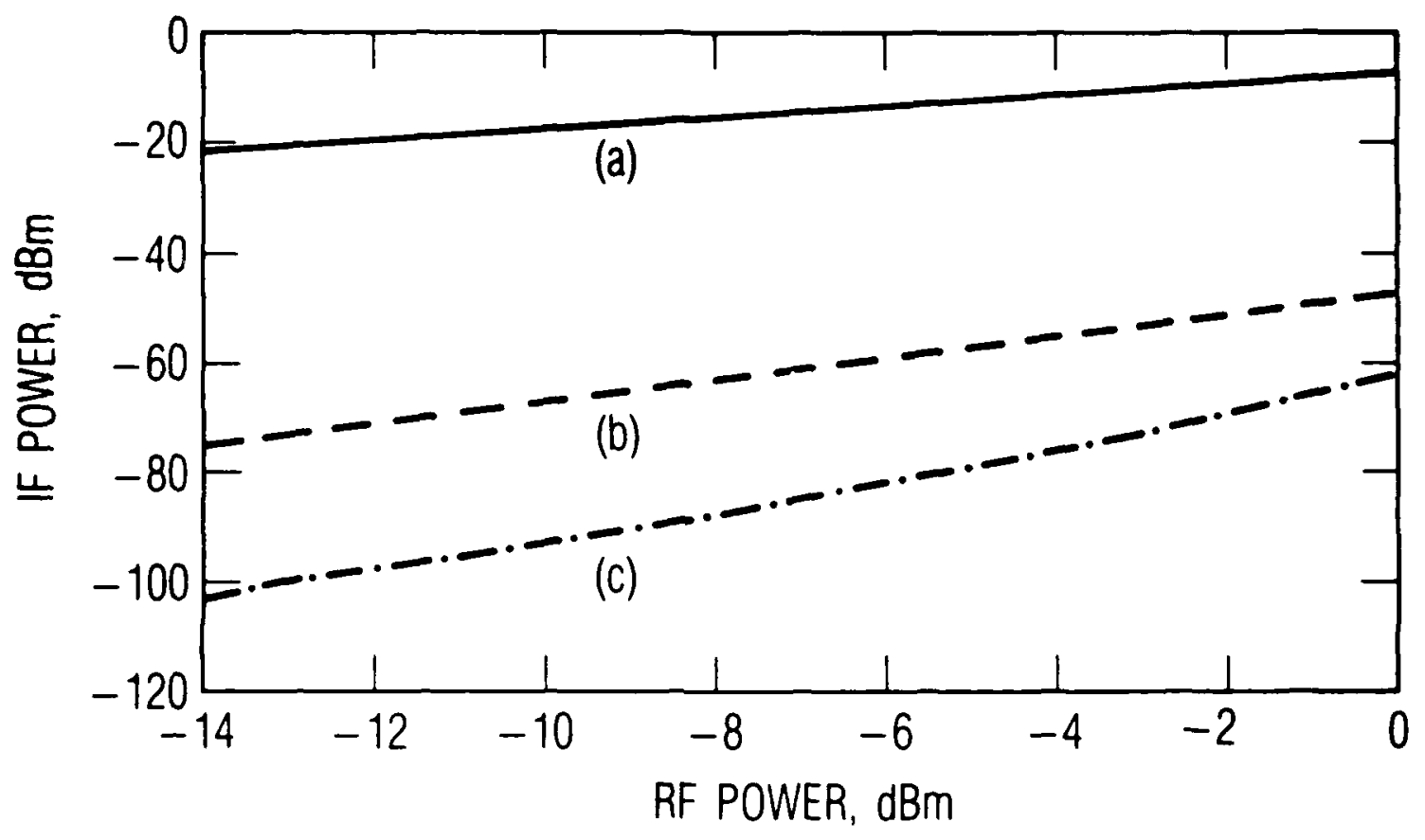

Fig. 6. (a) Linear, (b) Second-Order, and (c) Third-Order IM Levels vs. RF Level 
A simple balanced-mixer circuit that uses the resistive channel of a GaAs MESFET exhibits intermodulation performance that exceeds the state of the art for commercially available diode mixers. Other performance parameters, e.g., conversion loss, port impedances, and Lo sensitivity, are equal to or better than those of such dicde mixers. 


\section{REFERENCES}

1. J. H. Lepoff and A. M. Cowley, "Improved Intermodulation Rejection in Mixers," IEEE Trans. Microwave Theory Tech. MTT-14 [12], 618-623 (December 1966).

2. C. P. Tou and B. C. Chang, "A Technique for Intermodulation Reduction in Mixers," IEEE Symp. Electromagnetic Compatibility Digest, 128-132 (1981).

3. L. M. Kushnir, V. V. Soina, M. S. Fogel'son, and E. N. Gusinskiy, "Influence of Diode and Load Parameters on Intermodulation Noise Suppression in a Diode Mixer," Telecomm, and Radio Eng. 33 [3], 118-120 (March 1978).

4. J. G. Gardiner and A. M. Yousif, "Distortion Performance of Single-Balanced Diode Modulators," Proc. IEEE 117 [8], 1609-14 (August 1970). 
The Aerospace Corporation functions as an "archicect-engineer" tor national security projects, specializing in advanced military space systems. Providing research support, the corporation's Laboratory operations conducts experimental and theoretical investigations that focus on the application of sclentific and technical advances to such systems. Vital to the success of these investigations is the technical staff's wide-ranging expertise and its ability to stay current with new developments. This expertise is enhanced by a research program almed at dealing with tie many problems associated with rapidly evolving space systems. Contributing their capabilities to the resedrch effort are these individual laboratories:

Aerophysics Laboratory: Launch vehicle and reentry tluid mechanics, heat transfer and fighe dynamics; chemical and electric propulsion, propellant chemistry, chemical dynamics, environmental chemistry, trace detection; spacecraft structural mechanics. contamination, chermal and structural control; high temperature thermomechanlcs, gas kinetics and radiation; $c w$ and pulsed chemical and excimer laser development including chemfcal kinetics, spectroscopy, optical resonators, beam curtrul, atmospheric propagation, laser effects and colntermeasures.

Chemistry and Physics Laboratory: Atmospheric chemical reactions, atmospheric optics, light scattering. state-spectflc chenical reactions and radiative signatures of missile plumes, sensor out-of theld-of -view rejection, applied laser spectroscopy, laser chemistry, laser opcoelectronics, solar cell physics, battery electrochemistry, space vacuum and radlation effects on materials, Iubrication and surface phenomena, thermionic emission, photosensitive materials and detectors, atomic frequency standards, and environmental chemistry.

Computer Sclence Laboratory: Program verification, program translation, performance-sensitive system design, distributed architectures for spaceborne computers, fault-tolerant compucer systems, artificial intelligence, microelectronics applications, communication ptotocols, and computer security.

Electronics Research Laboratory: Mictoelectronics, solld-state device physics, compound semiconductors, radiation hardening; electroroptics, quantum electronics, solid-state lasers, optical propagation and communications; microwave semiconductor devices, microwave/millimeter wave measurements, diagnostics and radiometry, microwave/millimeter wave thermionic devices; atomic time and frequency standards; antennas, if systems, electromagnetic propagation phenomena, space communication systems.

Materials Sciences Laburatory: Development of new materials: metals, alloys, ceramics, polymers and their composites, and new forms of carbun; nun destructive evaluation, component raflire analysis and reliability; fracture mechanics and stress corrosion; analysis and evaluation of materials at cryogenic and elevated temperatures as well as in space and enemy-induced environments.

Space Sciences Laboratory: Magnetospheric, auroral and cosmic ray physics, wave-particle interactions, magnetospherte plasma waves; atmospheric and lonospheric physics, density and composition of the upper atmosphere, remote sensing using atmospheric radiation; solar pinsics, infrared astronomv. infrared signature analysis; effects of solar activity, mistnet ic storms and nuclear explosions on the earth's atmosphere, ionosphere and magnetosphere: effects of electromagnetic and particulate raviations on space systems: space instrumentation. 


$$
\begin{aligned}
& E N D \\
& 10-81 \\
& D T I C
\end{aligned}
$$

